\title{
Alpine vegetation along multiple environmental gradients and possible consequences of climate change
}

\author{
Sølvi Wehn • Sverre Lundemo • Jarle I. Holten
}

Received: 15 January 2014/Accepted: 13 August 2014/Published online: 23 August 2014

(C) The Author(s) 2014. This article is published with open access at Springerlink.com

\begin{abstract}
Alpine plant communities are being subject to climate change, resulting in the need for plants to adapt to new conditions. In this study, we investigated changes in vascular plant diversity along a coast-inland gradient in central Norway. At four different mountains, species were registered in $100 \mathrm{~m}^{2}$ plots, laid out from the forest line to the summit. In addition, plots were grouped into snow cover duration (short, medium, and long) and aspect (North, South, East, and West). We documented richness and spatial turnover of species and functional groups (trees and tall shrubs, dwarf shrubs, herbs, graminoids, and seedless plants) along the different gradients using glm and RDA. Thereafter, we included variables from climate scenario models in the derived glm's to estimate how species richness and the distribution of these groups could be impacted by future climate change. Our results show that there are significant changes in species and functional groups along the regional gradient. Further, also local
\end{abstract}

This article is part of the special issue Vegetation in cold environments under climate change.

Electronic supplementary material The online version of this article (doi:10.1007/s00035-014-0136-9) contains supplementary material, which is available to authorized users.

S. Wehn $(\bowtie) \cdot$ S. Lundemo

Grassland and Landscape Division, Bioforsk, Norwegian Institute for Agricultural and Environmental Research,

Kvithamar, 7512 Stjordal, Norway

e-mail: solvi.wehn@bioforsk.no

S. Lundemo

Department of Ecology and Genetics, Uppsala University,

75236 Uppsala, Sweden

J. I. Holten

Terrestrial Ecology Research, 7350 Buvika, Norway variation due to altitude and snow cover duration influenced species distribution and composition. Predictions of future vegetation showed an increase in species richness compared to present, with most changes occurring in areas of long and medium snow cover duration. We predicted the main changes to occur at lower altitudes. Here, dwarf shrubs and graminoids were expected to increase the most in number of species, whereas species of trees and tall shrubs were expected to increase the most in proportion.

Keywords Alpine plants · Coast-inland .

Functional groups $\cdot$ Monitoring $\cdot$ Climate change

\section{Introduction}

Alpine environments are changing, both due to changes in land use and climate. The effects of climate change can occur as direct changes in abiotic factors like temperature and precipitation levels, as well as indirect changes due to, e.g. changed biotic interactions (Dullinger et al. 2004; Olsen and Klanderud 2013). Temperature change can lead to increased stress in plants, e.g. reduced tissue water status or changed photosynthesis efficiency, which can affect internal processes at different life stages (Wahid et al. 2007). This can impact the distribution and abundance of plants, though the effects are likely to vary between species (see, e.g. Parmesan 2006 and references therein). Consequences of climate change for biodiversity, both in terms of communities and species, have been subject to a number of studies (see, e.g. Parmesan and Yohe 2003; Vellend et al. 2013, and references therein), and plants can address such changes through phenotypic plasticity, genetic adaptation or migration (Hoffmann and Sgrò 2011). Of these, Parmesan (2006) points to range shifts (migration) as being the 
more important response. Thus, the rate at which populations are able to track climate change can be crucial for long-term persistence. However, for populations occurring in marginal habitats, any of these options can be hard to achieve, increasing the risk of local extinction. At the same time, changed conditions can also facilitate immigration and establishment of new species. Changed environmental conditions can thus result in either a net increase, decrease or no change in species diversity (Vellend et al. 2013). Additionally, functional groups may have differential responses to change. A group that is expected to increase in coverage is dwarf shrubs (Sturm et al. 2001; Klanderud and Birks 2003), an expansion process that can take place through in-filling of existing areas, increased growth of existing individuals, and colonisation of new areas (Tape et al. 2006). Experimental warming studies show that in addition to dwarf shrubs, graminoids may increase, whereas lichens and mosses may decrease, though this effect will have pronounced regional variation (Walker et al. 2006; Elmendorf et al. 2012).

A number of recent studies have documented Europewide changes in alpine plant community composition, though there is clear spatial variation in the effects, both regionally and locally (Engler et al. 2011; Gottfried et al. 2012; Pauli et al. 2012; Elumeeva et al. 2013). Furthermore, this trend is projected to continue in the future, with stronger impacts in areas where increased temperature coincides with reduced precipitation (Engler et al. 2011). Thus, it is of interest to document how different plant communities respond to such environmental changes. Changes in temperature and precipitation patterns have been documented from Norwegian mountains (Hanssen-Bauer 2005; Isaksen et al. 2007). Accordingly, there have been several studies focusing on how alpine vegetation responds to physical change. Klanderud and Birks (2003) resurveyed (68 years after the initial survey) several mountain peaks in Jotunheimen, and found increased species richness in 19 out of 23 peaks. Similarly, Odland et al. (2010) resurveyed 13 mountain summits in a mountain area south of Jotunheimen (37 years after the initial survey) and documented an increase in species richness on all summits. Michelsen et al. (2011) found changes in vegetation composition at three out of four summits in a study from Dovrefjell (7 years after the initial survey), but only one of the summits showed an increase in species richness. According to Gottfried et al. (2012), the Dovrefjell mountain region differed from other European mountains in rate of vegetation change, being the only region where thermophilous species had not increased. The degree of climate change has been relatively moderate in this region compared to other European regions (Michelsen et al. 2011; Wehn et al. 2012), as well as compared to other mountain regions along the coast-inland transect in central Norway (Tveito and Dyrrdal 2011).
Gradients of moisture, snow cover duration and temperature are some of the key abiotic drivers of alpine plant species distribution. These gradients are considered multiscalar; e.g. at meso-scale the gradients define the lower limits of the alpine zone and at micro-scale the gradients define micro refuges (Carlson et al. 2013). Even though several studies have already documented vegetation change in several mountain areas (Odland et al. 2010; Michelsen et al. 2011; Gottfried et al. 2012), we have little knowledge about the joint effect of the multi-scalar abiotic drivers on vegetation distribution. Thus, there is a need to learn more about the effect of these multiple and multi-scalar factors on vegetation to establish cognition about the potentials for migration and local extinctions in changing climates.

We, therefore, examined alpine vegetation changes along four abiotic gradients: (1) oceanicity, (2) altitude, (3) snow cover duration, and (4) aspect in central Norway. More specifically, we investigated how species, species richness, functional groups and functional group abundance of vascular plants were distributed along and influenced by these four gradients. Finally, based on this information, we made predictions regarding how climate change may affect plant diversity within the study area.

\section{Materials and methods}

Study area and sampling design

We established in total 113 permanent plots $(10 \times 10 \mathrm{~m})$ in four mountains along a coast-inland gradient in central Norway (Table 1). The mountains were the coastal Stortussen, the inner fiord mountain Kaldfonna, the mountain Kolla at the main watershed at Dovrefjell, and the easternmost mountain Tron (Fig. S1). The mountains mainly consist of gneiss and thus display relatively species poor vegetation. However, the easternmost mountain also include richer bedrocks such as gabbro, schist and amphibolite (data obtained from NGU; http://geo.ngu.no/kart/berggrunn). Domestic sheep (Ovis aries) are range grazing in all study areas and in Kolla also wild reindeer (Rangifer tarandus) and muskox (Ovibos moschatus) are present.

The permanent plots were established as part of a project aiming to monitor vegetation changes in mountains due to predicted climate changes (GLORIA Norge; http://www. geo.uib.no/gloria/). Our study was based on the baseline vegetation data sampled in 2009-2012, with one mountain being sampled each year. In contrast to the well-known GLORIA multi-summit approach, plots were distributed in a systematic way through stratification of mountain slopes from the forest line upwards to the summit in 100 meter vertical belts on each mountain (Fig. S2). In each belt, 1-3 plots were located, covering the local snow cover duration 
Table 1 (a) Locality name, coordinates, max altitude, forest line, and number of plots $\left(100 \mathrm{~m}^{2}\right)$ established at each mountain included in the study, (b) normals (mean of range) and predicted future climate change (based on data obtained from NVE Norwegian Water

\begin{tabular}{|c|c|c|c|c|c|c|c|c|}
\hline (a) & \multicolumn{2}{|l|}{ Stortussen } & \multicolumn{2}{|l|}{ Kaldfonna } & \multicolumn{2}{|l|}{ Kolla } & \multicolumn{2}{|l|}{ Tron } \\
\hline Coordinates (UTM) & \multicolumn{2}{|l|}{$62^{\circ} 52^{\prime} \mathrm{N}, 7^{\circ} 30^{\prime} \mathrm{E}$} & \multicolumn{2}{|l|}{$62^{\circ} 37^{\prime} \mathrm{N}, 8^{\circ} 38^{\prime} \mathrm{E}$} & \multicolumn{2}{|c|}{$62^{\circ} 17^{\prime} \mathrm{N}, 9^{\circ} 29^{\prime} \mathrm{E}$} & \multicolumn{2}{|c|}{$62^{\circ} 10^{\prime} \mathrm{N}, 10^{\circ} 41^{\prime} \mathrm{E}$} \\
\hline Altitude (m asl) & 1,027 & & 1,849 & & 1,651 & & 1,665 & \\
\hline Forest line (m asl) & Ca. 550 & & Ca. 840 & & Ca. 1,100 & & Ca. 94 & \\
\hline \# Plots & 16 & & 40 & & 37 & & 20 & \\
\hline (b) & Normal & Future & Normal & Future & Normal & Future & Normal & Future \\
\hline Precipitation (mm/year) & 3,500 & $\approx 3,700$ & 2,500 & $\approx 2,600$ & 875 & $\approx 900$ & 875 & $\approx 900$ \\
\hline Temperature (mean ${ }^{\circ} \mathrm{C} /$ year) & 5 & $\approx 5.5$ & 3 & $\approx 3.5$ & $\div 2.5$ & $\approx 1.9$ & $\div 2.5$ & $\approx 1.9$ \\
\hline Snow cover (days/year) & 175 & $\approx 150$ & $>250$ & $\approx 250$ & $>250$ & $\approx 200$ & 225 & $\approx 250$ \\
\hline
\end{tabular}

gradient. Whenever possible, vertical belts were located in different aspects. Furthermore, when locating the plots, the three vegetation classes, snow bed, leeside and ridge, were used as indices of snow cover duration. Plots located in snow bed vegetation, leeside and ridge were defined as being influenced by long, medium and short snow cover duration, respectively. Altitude was measured in each plot with a barometric altimeter and aspect was measured with a compass. Additionally, aspect was grouped into four classes (North, South, East, West) in the analyses. Finally, in each plot all vascular plant species present were registered and grouped in five functional groups: dwarf shrubs, graminoids, herbaceous, trees and tall shrubs, and seedless plants.

\section{Statistical analyses}

To analyse vascular plant species distribution along the four abiotic gradients (oceanicity, altitude, snow cover duration, aspect), we studied the distribution of species richness and functional group abundance ( $\alpha$-diversity of species and functional groups), as well as spatial turnover of the species and the functional groups ( $\beta$-diversity of species and functional groups). Richness and spatial turnover of species were based on presence/absence data of the registered species in each plot. Abundance and spatial turnover of functional groups were based on number and proportion of species in functional groups in each plot, respectively.

As a measure of $\beta$-diversity, we interpreted the lengths of DCA (Detrended correspondence analysis) axes (Lepš and Šmilauer 2003; Tuomisto 2010). The advantage of this approach is that the axes are expressed in standard deviation units (SD), which make comparisons across data sets possible (Feilhauer and Schmidtlein 2009; Tuomisto 2010). If $\mathrm{SD}>4$, there is a complete spatial turnover in the data set. The use of DCA axis length as a measure of $\beta$-diversity has been criticized as it is not a function of effective spatial turnover (Tuomisto 2010), but since we were mainly
Resources and Energy Directorate, www.senorge.no) and values of the explanatory variables in the climate scenario (20 years in future) used for predicting future species richness 
polynomial relations between the different $\alpha$-diversity measures and altitude. However, due to the complexity of the models and relatively low number of plots (n), only the linear relation between altitude and $\alpha$-diversity was included in the modelling procedures. In analyses of both $\beta$ - and $\alpha$ diversity, interaction effects between the environmental gradients were tested for using the procedures described above. To account for type I errors in the forward selection procedures, significance levels were adjusted with Bonferroni correction ( $\alpha / N c$, Lepš and Šmilauer 2003), reducing the significance level to $0.004(N \mathrm{c}=12)$.

CANOCO Version 4.55 (Ter Braak 1988-2006) was used to calculate $\beta$-diversity, perform partial RDA's and multivariate $F$-ratio tests, and $R 2.14 .2$ (http://www.r-project.org/) to compute glm's and likelihood ratio Chi-square tests.

Predicting future plant diversity changes

To predict distribution of future plant species richness and functional groups, we generated new values of the local predictors (altitude, snow cover duration and aspect). We added these "new" values to the models that included the predictors which best described variation in species richness and functional group distribution (based on the forward selection process described above).

Normals and future levels of precipitation, temperature and snow cover duration in the four mountains were obtained from NVE (Norwegian Water Resources and Energy Directorate; http://www.senorge.no/?p=klima; Table 1). The Normals were based on interpolated data from weather stations and the scenarios on downscaling of global climate models (Engen-Skaugen et al. 2008): all grids of $1 \mathrm{~km}$ resolution. As the NVE scenarios are coarse, we could not extract environmental measures directly to our study plot scale, and the scenarios were instead used as a basis to generate "new" variables.

There are a number of climate variables that shift along the altitudinal gradient within each mountain, such as temperature, humidity, and solar radiation. However, we based our predictions on the general decrease of temperature along the altitudinal gradient (according to the International standard atmosphere: $-0.65{ }^{\circ} \mathrm{C}$ per $100 \mathrm{~m}$ ) only. The "new altitude" of the plots was based on the formula: Altitude $+\frac{\text { (Normal-Future temperature) }}{0.065}$; see Normal and Future temperatures in Table 1. Remote sensing based on satellite images can give fine scale information on snow cover duration (see, e.g. Dirnböck et al. 2003) and thereafter be extrapolated to future scenarios. However, this approach was not chosen in our study due to lack of such data. In general, NVE predictions showed decreased snow cover duration. We simplified and assigned (by random) half of the plots in each mountain currently having long snow cover duration to have medium snow cover duration in the future.
Finally, we found it unlikely that the oceanicity gradient (the four mountains) would be defined by one climate variable only, but we assumed precipitation to be the main determinant. To estimate the effect of changed precipitation, we visually interpreted figures of the predicted changes based on the "new" variables described above ("new altitude" and "new snow cover duration") in each mountain separately, compared these and then assessed the final future trends.

\section{Results}

Changes along climatic gradients

At the regional level, alpine vascular plant diversity $(\gamma$ diversity) showed a total richness of 204 species, of which one taxon was registered at family level, seven at genus level, two as hybrids, and the rest at species level. There was a high but not complete species spatial turnover ( $\beta$-diversity) along the environmental gradients, with the length of dca axis 1 being $3.350 \mathrm{SD}$ and of dca axis 2 being 3.134 SD. Functional spatial turnover was small, both when based on number (length of dca axis 1: $1.129 \mathrm{SD}$ and length of dca axis 2: $1.481 \mathrm{SD}$ ) and proportion of species in functional groups (length of dca axis 1: $1.273 \mathrm{SD}$ and length of dca axis 2: 1.432 SD). Mean species richness ( $\alpha$-diversity) was 23.44 (range 3-57).

All climatic variables except aspect were included in the final models that best explained the variability of the diversity measures investigated. However, the relation with the predictors varied among the diversity measures (Table 2).

Species spatial turnover along the altitudinal gradients in the mountains varied among plots influenced by different snow cover duration (Fig. 1). Changes in species composition along the gradients were, however, not so evident in and between the two coastal mountains. Species spatial turnover was higher along the altitudinal gradient as well as between classes of different snow cover duration in the two inland mountains compared to the two coastal mountains. Further, among plots influenced by long snow cover duration, species composition change along the altitudinal gradient was lower compared to between plots influenced by short and medium snow cover duration.

Both measures of functional spatial turnover showed the highest change in Kaldfonna, the inner fiord mountain, and lowest change in Stortussen, the coastal mountain. Functional turnover in space based on number of species in each group increased along the altitudinal gradient and was higher in plots influenced by long snow cover duration compared to plots influenced by shorter snow cover duration. Functional turnover in space based on proportion of species in each functional group, on the other side, was highest at intermediate altitudes. 
Table 2 The linear predictor variables in models that best explained the variability of (a) $\beta$-diversity measures based on a forward selection procedure using multivariate $F$ ratios test of models derived from RDA (Redundancy Analyses), and (b) $\alpha$-diversity measure based on a forward selection procedure using likelihood ratio Chi-square tests of glm's (generalized linear models)
The predictors in the models are ordered due to their conformity
Model

Explained

variation $(\%)$

(a) $\beta$-diversity measures (spatial turnover)

Species

Functional groups

Number of species in functional groups

Proportion of species in functional groups

(b) $\alpha$-diversity measures

Species richness

Number of species of

Graminoids

Herbs

Trees and tall shrubs

Dwarf shrubs

Seedless plants

Proportion of species of

Graminoids

Herbs

Trees and tall shrubs

Dwarf shrubs

Seedless plants
Mountain $x$ altitude

51

$x$ snow cover duration

$$
\begin{aligned}
& \text { Altitude }+ \text { mountain } \\
& + \text { snow cover duration } \\
& \text { Mountain }+ \text { altitude }
\end{aligned}
$$

Altitude $\times$ snow cover duration

ns

Altitude $\times$ snow cover duration $\quad 36$

Altitude $\times$ mountain 63

Altitude + mountain $\quad 58$

Mountain + snow cover duration $\quad 30$

Mountain + altitude $\quad 41$

ns

Mountain + altitude 39

Mountain + snow cover duration $\quad 30$

Mountain

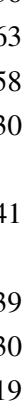

19

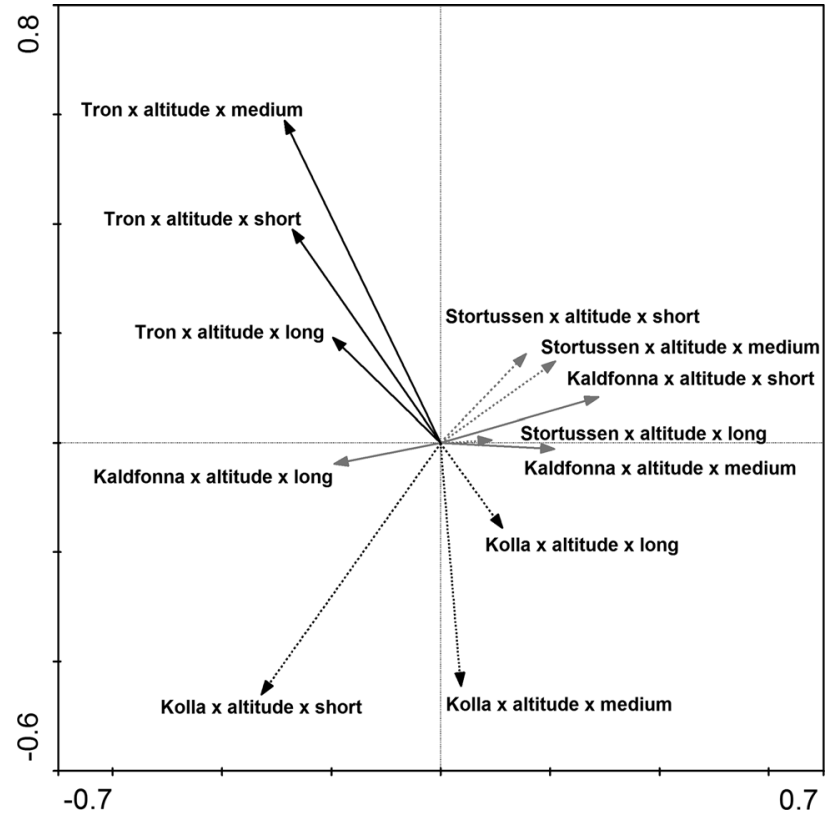

Fig. 1 Ordination diagram showing the RDA scores of the three-way interactions between environmental variables that significantly influenced species distribution (based on presence/absence data) in alpine vegetation zones in the central Norwegian study region. The environmental variables were altitude ( $\mathrm{m}$ asl), oceanicity (Stortussen, Kaldfonna, Kolla, Tron; listed from coast to inland) and snow cover duration (short, medium, long)
At lower altitudes, the highest species richness was observed in plots with medium and long snow cover duration (Fig. 2b). However, with increasing altitude richness decreased more in plots influenced by long snow cover duration compared to those influenced by medium snow cover duration. Richness in plots influenced by short snow cover duration decreased the least along the altitudinal gradient, and was similar to plots influenced by medium snow cover duration at higher altitudes. The relation between species richness and altitude was best explained by a third-order polynomial regression line; it decreased from the lowest altitudes to $800 \mathrm{~m}$ (asl), was constant until $1,200 \mathrm{~m}$, after which species richness declined abruptly (Fig. 2a).

The overall linear relations between numbers of herb-, tree and tall shrub- and dwarf shrub species and altitude were negative (Fig. S3a, b). However, the number of herb species was equal in all plots with short snow cover duration and decreased the most in plots influenced by long snow cover duration along the altitudinal gradient (Fig. S3b). Trees and tall shrubs became absent below the summits, except from in the most continental mountain. In this mountain, which also included the highest number of trees and tall shrubs, the decrease in species number in this group was not as steep as in the other mountains (Fig. S3a). The number of seedless plants was highest in this inland mountain (Fig. S3a) and in 
(a)

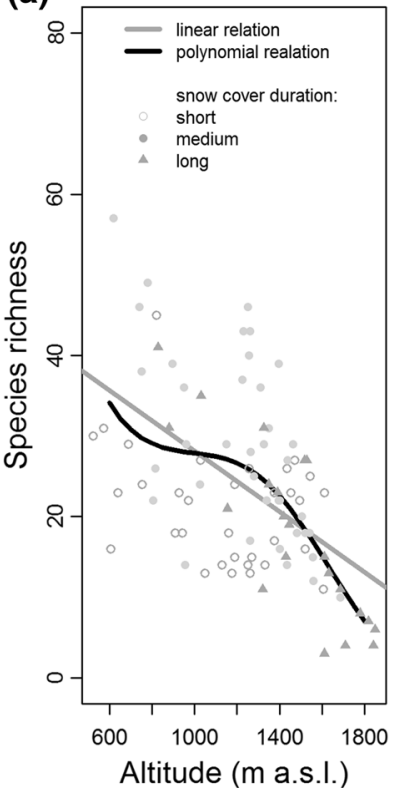

(b)

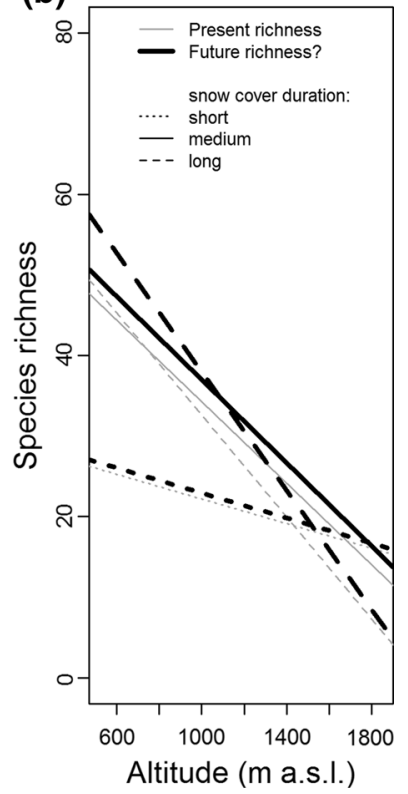

Fig. 2 Species richness along the altitudinal gradient. a The general linear trend and the significant polynomial relation. The study plots are sorted by snow cover duration. $\mathbf{b}$ The estimated present relation (grey) and the predicted future relation (black) in the three snow cover duration classes. Present richness is estimated by glm and predicted future richness is based on the glm that best described variation in species richness along climatic gradients in central Norway (see "Materials and methods") and climate scenarios derived from predicted values given by NVE (Norwegian Water Resources and Energy Directorate)

plots of medium snow cover duration (Fig. S3b). When comparing changes along the altitudinal gradient only, the number of herb and dwarf shrub species showed a secondorder polynomial relation (Fig. S4a, b). Both number of herb and dwarf shrub species increased from the lowest altitudes and peaked at $900 \mathrm{~m}$, whereas above this threshold, numbers decreased along the altitudinal gradient.

All but proportion of herb species differed among the mountains (Table 2; Fig. 3). The overall linear relation with altitude was positive with proportion of graminoid species and negative with proportion of tree and tall shrub species. However, the proportion of graminoids increased the most along the altitudinal gradient in the two mountains in the middle of the coast-inland gradient (Kaldfonna, Kolla), whereas proportion of trees and tall shrubs was constant along the altitudinal gradient in the most continental mountain (Tron) compared to the other mountains, where this group decreased abruptly. In addition, the overall proportions of graminoid- and dwarf shrubs species were lower, and the proportions of tree and tall shrub and seedless plant species were higher in Tron. Dwarf shrubs showed the highest proportion in plots influenced by short snow cover duration. When comparing changes along the altitudinal gradient only, proportion of graminoid species showed a second-order

polynomial relation (Fig. S4c). From the lowest altitudes to $1,100 \mathrm{~m}$, the proportion of graminoid species was constant; however, above this threshold, it increased with altitude.

Future plant diversity

In general, we predicted species richness to increase, with total mean richness being 25.98 (range 10-51) +2.54 species compared to the present. The highest increases in species richness were predicted in areas currently influenced by medium and long snow cover duration (Fig. 2b). In the areas retaining long snow cover duration, we predicted the increase in number of species to be low at the lower altitudes, and increasingly higher along the altitudinal gradient. In future areas with medium snow cover duration, we predicted number of species to increase at lower altitudes, but decrease at higher altitudes.

We also predicted a general increase in species number in each functional group except seedless plants (Fig. S3a, b). Changes in proportions of the functional groups, however, were not that prominent (Fig. 3). Numbers and proportion of species in functional groups were predicted to experience the largest changes at lower altitudes and in the two mountains in the middle of the coast-inland gradient (Figs. S3a and 3). If changes in numbers of species in functional groups were predicted, these were largest in areas influenced by long snow cover duration (Fig. S3b). Trees and tall shrubs were predicted to increase the most in proportion, especially at lower altitudes, except in the most continental mountain (Fig. 3). In this mountain, the only predicted change was a small increase in proportion of dwarf shrubs at the expense of proportion of herb species. Following the predictions visualized in the figures based on temperature- and snow cover duration scenarios, the most "stable" functional group would be seedless plants. In addition, the future vegetation will also be influenced by the estimated higher precipitation levels (Table 1). We, therefore, predict that vegetation in the inland mountains will become more similar to the present vegetation in the neighbouring mountain closer to the coast in the coast-inland gradient of the study region.

\section{Discussion}

To predict reliable future vegetation changes, multifactorial determinants have to be considered. We found that alpine plant species diversity was driven by multiple and interacting determinants; both locally (altitude and snow cover duration) and regionally (oceanicity). In addition, we found that effects of determinants varied between different diversity measures.

In our study, species richness showed a general negative relation with increasing altitudes, as expected in an 

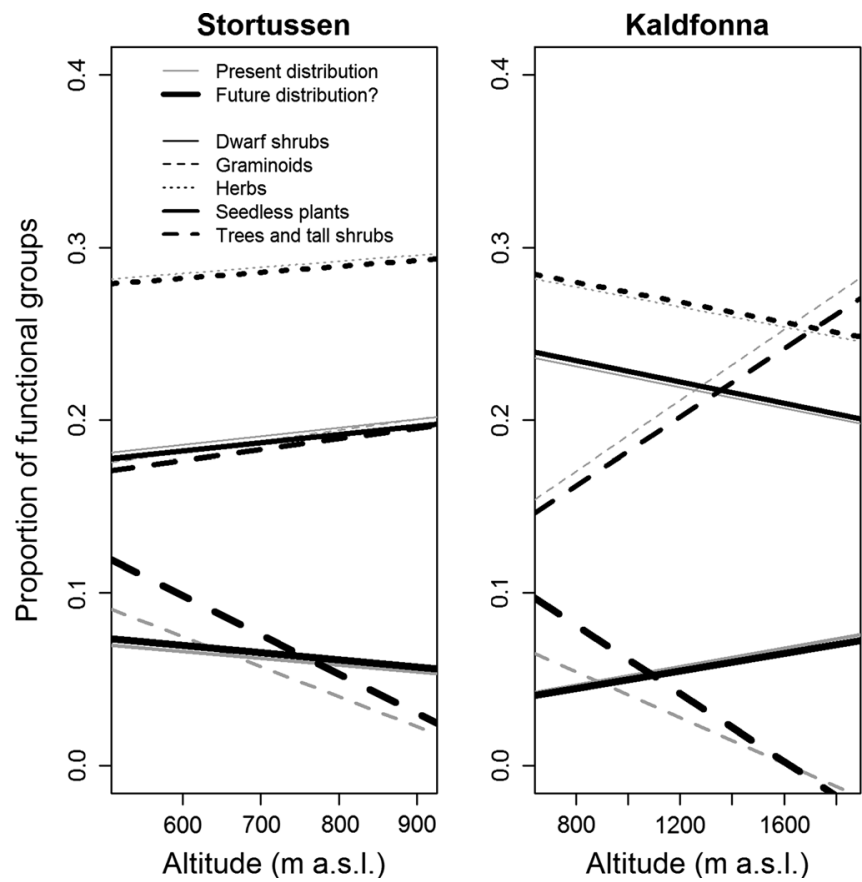

Fig. 3 Distribution of plant functional groups (based on proportion of species in each group) along the altitudinal gradient in four mountains (from coast to inland). Present distribution is shown as grey lines and predicted future distribution in a warmer climate as black lines.

increasingly harsher environment (see, e.g. Fosaa 2004). However, more detailed examinations of the relation between species number and altitude showed an abrupt decrease just above the forest line. The number of species was equal in plots at $800-1,200 \mathrm{~m}$ (Fig. 2a), which might be due to the presence of forest sink populations in this zone (Grytnes et al. 2006). Hence, climate change might cause more rapid shifts in the lower compared to higher zones. This strengthens our predicted trends of more change at lower altitudes. In this currently low alpine zone, forest species will expand and outcompete smaller light demanding alpine plants which will thus also expand upwards, if possible. The zone between the tipping point and the summit (above 1,200 $\mathrm{m}$ asl) can be narrow. When predicting future consequences of climate change, knowledge about this upper tipping point may give deeper insight into potential threats. The niche of alpine species will become narrower, especially those adapted to the harsher environment at the summits. As already documented in more southern European mountains (Gottfried et al. 2012), the final result will potentially be higher extinction at higher altitudes compared to our predicted future vegetation distributions.

In addition to varying effects along local gradients in different areas (mountains), our study also showed interaction effects within mountains. Felde et al. (2012) found an upward elevation expansion of species dependent upon long snow duration in a mountain area in central Norway, likely a
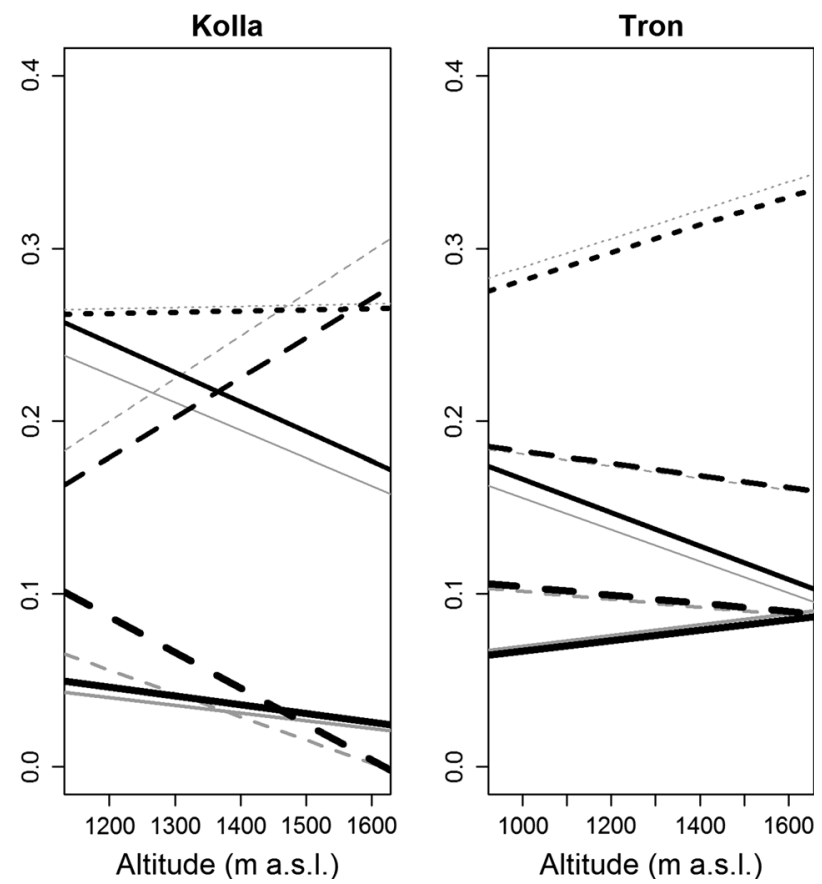

Present distribution is estimated by $\mathrm{glm}$ and predicted future distributions are based on the glm's (see "Materials and methods") and climate scenarios derived from NVE (Norwegian Water Resources and Energy Directorate)

response to increased precipitation at high elevations. As the climate scenarios used as basis for our predictions were coarse, we did not include varying changes in snow cover duration along the altitudinal gradient (discussed in the "Materials and methods" section). Snow cover depth and duration have been reported to have a significant effect on species richness, with the highest levels at intermediate levels (Chen et al. 2008; Carbognani et al. 2012), as was also shown in our study. However, when predicting future vegetation distribution, our results showed that at altitudes above $1,200 \mathrm{~m}$, species richness decreased compared to current richness in areas of medium snow cover duration, which may indicate a higher risk for species requiring a certain moisture level. Lenoir et al. (2013) showed how fine grained thermal variation was influenced not only by elevation and roughness (that can be assigned to our three snow cover duration classes; snow beds influenced by long snow cover duration are located in concave depressions, leesides influenced by medium snow cover duration are located in more or less plain slopes, ridges influenced by short or no snow cover duration are located in convex mounds), but also variables related to aspect. Horizontal migration along mountain slopes to other aspects might cause spatial buffering of regional vegetation responses to climate change, which is estimated to be relevant at these latitudes due to terrain roughness (Lenoir et al. 2013). Aspect was not included in our predictions as it was not considered 
significant. However, we may have underestimated the future effects of aspect due to unbalanced design.

Environmental variables do not influence different biodiversity measures equally. Löffler and Pape (2008) discussed the importance of the influence of several environmental factors as well as their interaction effects on species diversity, and found microclimate conditions to be more important than regional conditions in alpine tundra vegetation in Northern Norway. We found the same pattern in our more southern study region, but only when examining species richness. The effect of mountain and the relative amount of shifts in space explained by this regional predictor was high when examining all $\beta$-diversity measures as well as numbers in and proportions of functional groups. The coastinland gradient is considered the most important environmental gradient in Norway, but fewer environmental shifts along this gradient may result in a lower contribution to variation in species diversity in Northern Norway (Moen 1999; Bakkestuen et al. 2008; Fig. S1).

Vegetation patterns are, in addition to abiotic factors, influenced by inter- and intraspecific interactions. Following our predictions, trees and tall shrubs should show the highest increase at lower altitudes. This is as expected since the forest line, which in the absence of herbivory or logging is defined mainly by temperature, moves upward as temperature increases. Different competitive ability among species during the recruitment phase may affect future range dynamics in alpine environments (Dullinger et al. 2004), and it is known that trees and tall shrubs negatively affect smaller light demanding plants. On the other hand, competition has been shown to decrease community invasibility in warmer climates, with the establishment rate of invading species being high in areas with high graminoid species richness, and low in areas of high herb species richness (Olsen and Klanderud 2013). Thus, different levels of interspecific competition can result in varying levels of vegetation change in different vegetation classes. Therefore, as we registered high herb species richness in plots with both long and medium snow cover durations at lower altitudes, the large forecasted change in species number in areas with medium snow cover duration at these altitudes might be less marked. Graminoids dominated in areas of short snow cover duration at high altitudes, areas which, therefore, may show higher changes compared to our predictions.

When modelling and predicting vegetation shifts, it is important to not only examine species richness, but also relationships between species and functional groups. Venn et al. (2012) found that species numbers of shrubs and graminoids increased whereas number of herbs decreased at mountains in Australia. Similarly, Michelsen et al. (2011) found increased abundance of some shrubs and graminoids in Dovrefjell, Norway. In our study, we predicted higher species numbers in all functional groups except seedless plants. However, we only predicted proportions of tree and tall shrub- and dwarf shrub species to increase, whereas proportion of graminoid species was to decrease. As we included analyses of proportions, we found more balanced results. A shortcoming of our study, however, is that we did not investigate relative abundance, which would have provided more detailed knowledge.

An important factor influencing vegetation is land use, such as grazing by domestic animals (Löffler and Pape 2008; Wehn 2009; Wehn et al. 2011, 2012; Carlson et al. 2013). Sheep are grazing in all studied mountains, though densities in most of the study area were less than 25 sheep $/ \mathrm{km}^{2}$ (data from http://kilden.skogoglandskap.no/map/kilden/index. jsp). Such low densities have been shown unable to influence shifts in vegetation classes in the transition zone between boreal and alpine (Wehn et al. 2011). Grazing by domestic sheep may, however, influence the response of dwarf shrubs to climate warming. Evju et al. (2012) found woody species to be most vulnerable to sheep grazing, while Speed et al. (2013) found that expansion of woody Salix spp. to be constrained at higher elevations, with the opposite effect at lower elevations. The differences we observed might, therefore, also be attributed to varying influence by sheep grazing and thus also future vegetation change might experience different shifts compared to our predictions.

To forecast future alpine plant distributions Carlson et al. (2013) argued for the use of integrated models and long-term monitoring projects. This study is a first step in establishing a long-term monitoring project, where comparison of the vegetation along the multiple environmental gradients described will be used as a reference for future monitoring. Each plot has been equipped with a temperature logger which will enable later correlations between vegetation changes and temperature variation. Further, satellite images and soil moisture loggers will be used to register changes in snow cover distribution. Therefore, more fine-scaled climatic variables will be provided and thus improve some of the shortcomings of this study. In total, this will capture variation in vegetative response after habitat change, both at a local and regional scale and establish whether the predictions calculated in this study will be fulfilled.

\section{Conclusions}

We have shown the importance of including a number of climate gradients such as oceanicity, altitude, and snow cover duration when investigating alpine vegetation distribution. Species richness was mainly influenced by altitude and snow cover duration in mountains in central Norway. Species composition was also influenced by oceanicity, whereas proportions of species in functional groups were mainly influenced by oceanicity and altitude. Further, we 
have shown that by including these climate variables in predictions we have been able to estimate potential future alpine vegetation. The predictions showed the most rapid shifts in the low alpine zone due to upward migration of woody species; however, above an altitudinal threshold at approximately $1,200 \mathrm{~m}$, there might be a high potential risk of local species extinctions.

Acknowledgments Thanks to Anne Olga Syverhuset for help with field work and anonymous reviewers and editors for comments on earlier versions of the manuscript. Funding was provided by The Norwegian Environment Agency, the county councils of Møre og Romsdal, Oppland and Sør-Trøndelag, and the municipalities of Eide and Sunndal through the project GLORIA Norge, as well as Bioforsk, Norwegian Institute for Agricultural and Environmental Research.

Open Access This article is distributed under the terms of the Creative Commons Attribution License which permits any use, distribution, and reproduction in any medium, provided the original author(s) and the source are credited.

\section{References}

Bakkestuen V, Erikstad L, Halvorsen R (2008) Step-less models for regional environmental variation in Norway. J Biogeogr 35:1906-1922

Carbognani M, Petraglia A, Tomaselli M (2012) Influence of snowmelt time on species richness, density and production in a late snowbed community. Acta Oecol 43:113-120

Carlson BZ, Randin CF, Boulangeat I, Lavergne S, Thuiller W, Choler P (2013) Working toward integrated models of alpine plant distribution. Alp Bot 123:41-53

Chen W, Wu Y, Wu N, Luo P (2008) Effect of snow-cover duration on plant species diversity of alpine meadows on the eastern Qinghai-Tibetan plateau. J Mt Sci 5:327-339

Dirnböck T, Dullinger S, Grabherr G (2003) A regional impact assessment of climate and land-use change on alpine vegetation. J Biogeogr 30:401-417

Dullinger S, Dirnböck T, Grabherr G (2004) Modelling climate change-driven treeline shifts: relative effects of temperature increase, dispersal and invasibility. J Ecol 92:241-252

Elmendorf SC, Henry GHR, Hollister RD et al (2012) Global assessment of experimental climate warming on tundra vegetation: heterogeneity over space and time. Ecol Lett 15:164-175

Elumeeva TG, Onipchenko VG, Egorov AV et al (2013) Long-term vegetation dynamic in the Northwestern Caucasus: which communities are more affected by upward shifts of plant species? Alp Bot 123:77-85

Engen-Skaugen T, Haugen JE, Hanssen-Bauer I (2008) Dynamically downscaled climate scenarios available at the Norwegian Meteorological Institute-per December 2008 Met.no report no. $24 / 2008$

Engler R, Randin CF, Thuiller W et al (2011) 21 st century climate change threatens mountain flora unequally across Europe. Glob Change Biol 17:2330-2341

Evju M, Hagen D, Hofgaard A (2012) Effects of disturbance on plant regrowth along snow pack gradients in alpine habitats. Plant Ecol 213:1345-1355

Feilhauer H, Schmidtlein S (2009) Mapping continuous fields of forest alpha and beta diversity. Appl Veg Sci 12:429-439
Felde VA, Kapfer J, Grytnes J-A (2012) Upward shift in elevational plant species ranges in Sikkilsdalen, central Norway. Ecography 35:922-932

Fosaa AM (2004) Biodiversity patterns of vascular plant species in mountain vegetation in the Faroe Islands. Divers Distrib 10:217-223

Gottfried M, Pauli H, Futschik A et al (2012) Continent-wide response of mountain vegetation to climate change. Nat Clim Chang 2:111-115

Grytnes JA, Heegaard E, Ihlen PG (2006) Species richness of vascular plants, bryophytes, and lichens along an altitudinal gradient in western Norway. Acta Oecol 29:241-246

Hanssen-Bauer I (2005) Regional temperature and precipitation series for Norway: Analyses of time-series updated to 2004. Met. no report 15/2005-Climate. Norwegian Meteorological Institute, Oslo

Hoffmann AA, Sgrò CM (2011) Climate change and evolutionary adaptation. Nature 470:479-485

Isaksen K, Sollid JL, Holmlund P, Harris C (2007) Recent warming of mountain permafrost in Svalbard and Scandinavia. J Geophys Res 112 F02S04, doi:10.1029/2006JF000522

Klanderud K, Birks HJB (2003) Recent increases in species richness and shifts in altitudinal distributions of Norwegian mountain plants. Holocene 13:1-6

Legendre P, Anderson MJ (1999) Distance-based redundancy analysis: testing multispecies responses in multifactorial ecological experiments. Ecol Monogr 69:1-24

Lenoir J, Graae BJ, Aarrestad PA et al (2013) Local temperatures inferred from plant communities suggest strong spatial buffering of climate warming across Northern Europe. Glob Change Biol 19:1470-1481

Lepš J, Šmilauer P (2003) Multivariate analysis of ecological data using CANOCO. Cambridge University Press, Cambridge

Löffler J, Pape R (2008) Diversity patterns in relation to the environment in alpine tundra ecosystems of northern Norway. Arct Antarct Alp Res 40:373-381

Michelsen O, Syverhuset AO, Pedersen B, Holten JI (2011) The impact of climate change on recent vegetation changes on Dovrefjell, Norway. Diversity 3:91-111

Moen A (1999) National atlas of Norway: vegetation. Norwegian Mapping Authority, Hønefoss

Odland A, Høitomt T, Olsen SL (2010) Increasing vascular plant richness on 13 high mountain summits in Southern Norway since the early 1970s. Arct Antarct Alp Res 42:458-470

Olsen SL, Klanderud K (2013) Biotic interactions limit species richness in an alpine plant community, especially under experimental warming. Oikos 123:71-84

Parmesan C (2006) Ecological and evolutionary responses to recent climate change. Annu Rev Ecol Evol Syst 37:637-669

Parmesan C, Yohe G (2003) A globally coherent fingerprint of climate change impacts across natural systems. Nature 421:37-42

Pauli H, Gottfried M, Dullinger S et al (2012) Recent plant diversity changes on Europe's mountain summits. Science 336:353-355

Speed JDM, Austrheim G, Hester AJ, Mysterud A (2013) The response of alpine Salix shrubs to long-term browsing varies with elevation and herbivore density. Arct Antarct Alp Res 45:584-593

Sturm M, Racine C, Tape K (2001) Increasing shrub abundance in the Arctic. Nature 411:546-547

Tape K, Sturm M, Racine C (2006) The evidence for shrub expansion in Northern Alaska and the Pan-Arctic. Glob Change Biol 12:686-702

Ter Braak CJF (C) 1988-2006 Biometris - quantitative methods in the life and earth sciences Plant Research International, Wageningen University and Research Centre Box 100, 6700 AC Wageningen, the Netherlands 
Tuomisto H (2010) A diversity of beta diversities: straightening up a concept gone awry. Part 2. Quantifying beta diversity and related phenomena. Ecography 33:23-45

Tveito OE, Dyrrdal AV (2011) Climate and snow. In Holten JI and Aune EI (eds) Altitudinal distribution patterns of alpine plants. Studies along a coast-inland transect in southern Scandes, northern Europe. Tapir, Trondheim, pp 25-34

Vellend M, Baeten L, Myers-Smith IH et al (2013) Global metaanalysis reveals no net change in local-scale plant biodiversity over time. Proc Nat Acad Sci USA 110:19456-19459

Venn S, Pickering C, Green K (2012) Short-term variation in species richness across an altitudinal gradient of alpine summits. Biodivers Conserv 21:3157-3186

Wahid S, Gelani S, Ashraf M, Foolad MR (2007) Heat tolerance in plants: an overview. Environ Exp Bot 61:199-223
Walker MD, Wahren CH, Hollister RD et al (2006) Plant community responses to experimental warming across the tundra biome. Proc Nat Acad Sci USA 103:1342-1346

Wehn S (2009) A mapbased method for exploring responses to different levels of grazing pressure at the landscape scale. Agri Ecosyst Environ 129:177-181

Wehn S, Pedersen B, Hanssen SK (2011) A comparison of influences of cattle, goat, sheep and reindeer on vegetation changes in mountain cultural landscapes in Norway. Landscape Urban Plan 102:177-187

Wehn S, Olsson G, Hanssen S (2012) Forest line changes after 1960 in a Norwegian mountain region-implications for the future. Nor Geogr 66:2-10 